\title{
Synthesis of Some New Benzoxazepine Compounds Form Derivatives of Schiff Bases
}

\author{
Amena A. Ahmed ${ }^{1 *}$; Ahmad Kh. Ahmad ${ }^{2}$; Natiq G. Ahmed ${ }^{3}$ \\ Department of Chemistry, College of Education for Pure Science, University of Mosul, Mosul, Iraq \\ Email: ${ }^{1 *}$ altaeeamena@gmail.com, ${ }^{2}$ ahmad alkhayat@yahoo.com ${ }^{3}$ natiq.ahmad@yahoo.com \\ (Received January 07, 2019; Accepted February 27, 2019; Available online March 01, 2020) \\ DOI: 10.xxxxx/edusj.2020.xxxxxx.xxxx, () 2020, College of Education for Pure Science, University of Mosul. \\ This is an open access article under the CC BY 4.0 license (http://creativecommons.org/licenses/by/4.0/).
}

\begin{abstract}
:
This study involved synthesize $(1,3)$ oxazepine. The step 1 includes the preparation of compound (1), (5-Bromo-2-mercapto-6-(4-methoxyphenyl) pyrimidin-4(3H)-one), by the condensation of anisaldehyde and ethylbromoacetat and thiourea in EtOH. In the step2, chalcones (26 ) have been produced. The reaction of compound (1) with chalcones (2-6) that gives azo Michael adduct (7-11) is made in the third step. Schiff's bases (12-16) were prepared by the reaction ketones (7-11) with 2,4-dinitroaniline. Finally preparation of new benzo [1,3] oxazepine compounds (17-21) are prepared by the reaction of phthalic anhydride with Schiff's bases. The synthesized compounds are identified by physical (melting points, colour change) and spectral methods such as (IR, proton-nmr).
\end{abstract}

Keywords: $\beta$-amino ketones, Michael addition, Oxazepine

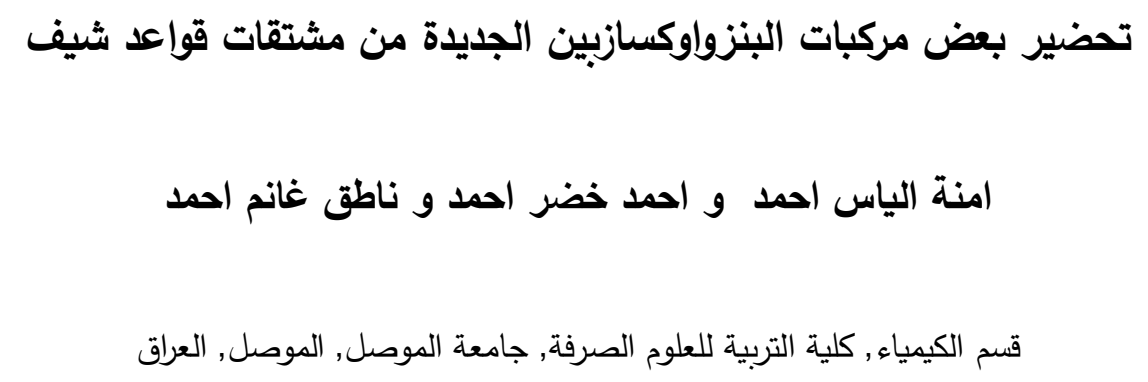

الخلاصة

الدراسة تضمنت تحضير (3,1) اوكسازبين. في الخطوة الاولى تم تحضير المركب (1) 5- برومو-2-ميركابتو - 6-

(4- ميثوكسي فنيل) بيرميدين - 3H) اون. بتكاثف الانيسالديهيد وايثايل برومو اسيتيت والثايوريا في الايثانول. الخطوة الثانية نتج

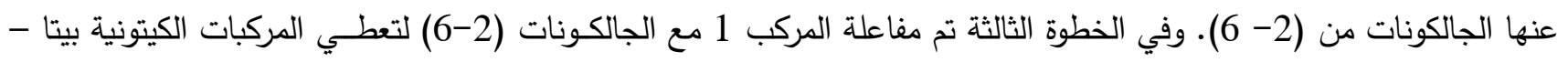

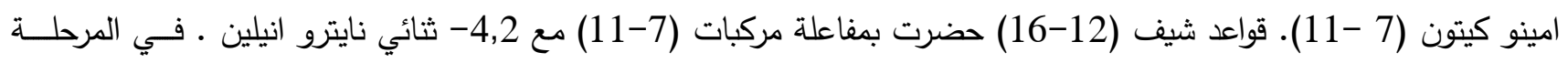

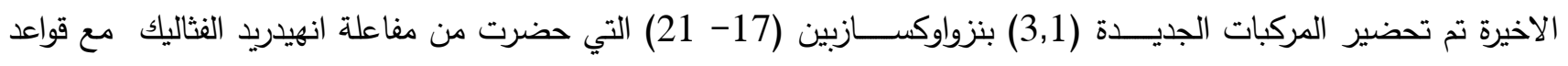

شيف. المركبات المحضرة شخصت بوساطة الخواص الفيزيائية (درجة الانصهار، اللون ). الطرق الطيفية ( الاشعة تحت الحمراء,

الرنين النووي المغناطيسي) 
الكلمات المفتاحية: بيتا - امينو كيتون, اضافة مايكل، اوكسازبين

\section{Introduction}

The Michael's addition includes the addition of nucleophile, also called a Michael donor that is added to an activated electrophilic olefin[1].The adducts of Michael reaction which are $\beta$-Amino ketones are important intermediates for the synthesis of a wide variety of compounds. The applications of this process of 1,4-addtion is the synthesis of $\beta$-amino Ketones; it can be done prepared throw the classical Mannich reaction, Therefor, a variety of methods appeared in the literature[2]. For synthesis of $\beta$-amino ketones, the Michael addition of nucleophilic compound to $\alpha, \beta$-unsaturated carbonyl compounds is convenient rote for the construction of $\mathrm{C}-\mathrm{C}$ and $\mathrm{C}-\mathrm{N}[3,4,5]$ bands. These compounds were used as starting materials for the synthesis of different heterocycles[6]. Schiff's bases, (R-CH=N$\mathrm{R} 1)$ where R and R1 are alkyl, aryl, cyclo alkyl or heterocyclic group) are products of primary amines with carbonyl compound like $\beta$-amino carbonyl compounds[7]. They show biological activities including antibacterial, antifungal[8,9], anticancer[10] and herbicidal active[11]. Oxazpine is nonhomologous seven membered ring that contains two heteroatoms (oxygen and nitrogen)[12]. Schiff's bases react with phthalic anhydride to give 1,3-Benzooxazpine[13].

\section{Experimental}

All reagents and chemicals are from BDH and Fluka, used without purification. Melting points that measured using: Electro thermal IA 9100 melting points apparatus type (not corrected). Shimadzu FT- IR-8400 used to get the ir spectra. Proton nmr were recorded on Bruker $400 \mathrm{MHz}$ spectrometer using acetone- $\mathrm{d}^{6}$ and $\mathrm{CDCl}_{3}$ as solvent in $\mathrm{UK}$, Leister University.

\section{Sythesis of (5-Bromo-2-mercapto-6-(4-methoxyphenyl) pyrimidin-4(3H)-one)}

Equimolar (0.001 mol) of anisaldehyde, ethylbromoacetate and thiourea were dissolved in abs. EtOH. Potassium carbonate $(0.003 \mathrm{~mol})$ is added to this reaction mixture and refluxed for $2 \mathrm{~h}$; the solvent was concentrated then poured in to ice cold water with stirring, neutralizion with glacial acetic acid[14].The solid produce filtered and washed with water as well as recrystallized from methanol. Table (1) involves physical properties.

\section{Synthesis of Chalcones (2-6)}

The mixture of substitute acetophenone and substitute benzaldehyde $(0.01 \mathrm{~mol})$ is dissolved in $(30 \mathrm{ml})$ ethanol, then added $(2 \mathrm{ml})$ to sodium hydroxide solution $40 \%$, stirring the mixture for (24 ) hours $[15,16]$. The ppt obtained was filtered, washed with water and recrystallized from ethanol, Table (1) involves physical properties.

\section{Synthesis of $\beta$-amino ketones compound (7-11)}

To $(0.01 \mathrm{~mol})$ of compound (1), (0.01 mol) of various chalcone, and $(2 \mathrm{ml}) \mathrm{NaOH} 30 \%$ were added to $(30 \mathrm{ml})$ of ethanol, stirred for $4 \mathrm{~h}$ on ice cold condition[17]. The solid formed is filtered and recrystallized from abs. EtOH Table (1) involves physical properties.

\section{Synthesis of Schiff's bases (12-16)}

A mixture of $\beta$-amino keton compounds (7-11) (0.01 mol) added to $(0.01 \mathrm{~mol})$ in abs. EtOH $(25 \mathrm{ml})$ containing a few amount of glacial acetic acid is stirring for $4 \mathrm{~h}[18]$ . The solvent was evaporated under vacuum. The solid yield is crystallized from methanol. Table (1) involves physical properties. 


\section{Synthesis of benzo[1,3] oxazepine derivatives (17-21)}

A mixture of Schiff's bases (12-16) (0.0004 mol) added to $(0.0006 \mathrm{~mol})$ of phthalic anhydride in $(20 \mathrm{ml})$ of abs. EtOH was refluxed for $6 \mathrm{~h}[19]$. The ppt was filtered,washed with water and recrystallized from ethanol. Table (1) involves physical properties.

Table(1) Some physical characteristic For comp. (1-21)

\begin{tabular}{|c|c|c|c|}
\hline $\begin{array}{c}\text { Comp. } \\
\text { No }\end{array}$ & Moleculer Formula \&M.Wt & m.p. ${ }^{\circ} \mathrm{C} \&$ Color & Yield \% \\
\hline 1 & $\begin{array}{c}\mathrm{C}_{11} \mathrm{H}_{9} \mathrm{BrN}_{2} \mathrm{O}_{2} \mathrm{~S} \\
313\end{array}$ & $\begin{array}{l}268-270 \\
\text { Yellow }\end{array}$ & 93 \\
\hline 2 & $\begin{array}{c}\mathrm{C}_{15} \mathrm{H}_{11} \mathrm{NO}_{3} \\
253 \\
253 \\
\end{array}$ & $\begin{array}{l}132-134 \\
\text { Yellow }\end{array}$ & 70 \\
\hline 3 & $\begin{array}{c}\mathrm{C}_{15} \mathrm{H}_{11} \mathrm{NO}_{3} \\
253\end{array}$ & $\begin{array}{l}129-131 \\
\text { Yellow }\end{array}$ & 61 \\
\hline 4 & $\begin{array}{c}\mathrm{C}_{15} \mathrm{H}_{12} \mathrm{O} \\
208\end{array}$ & $\begin{array}{l}\text { 124-126 } \\
\text { Yellow }\end{array}$ & 85 \\
\hline 5 & $\begin{array}{c}\mathrm{C}_{17} \mathrm{H}_{16} \mathrm{O}_{2} \\
252 \\
\end{array}$ & $\begin{array}{c}180-182 \\
\text { Light Green }\end{array}$ & 71 \\
\hline 6 & $\begin{array}{c}\mathrm{C}_{15} \mathrm{H}_{11} \mathrm{NO}_{3} \\
253\end{array}$ & $\begin{array}{c}84-86 \\
\text { Brown }\end{array}$ & 75 \\
\hline 7 & $\begin{array}{c}\mathrm{C}_{26} \mathrm{H}_{20} \mathrm{BrN}_{3} \mathrm{O}_{5} \mathrm{~S} \\
566\end{array}$ & $\begin{array}{l}248-250 \\
\text { Yellow }\end{array}$ & 66 \\
\hline 8 & $\begin{array}{c}\mathrm{C}_{26} \mathrm{H}_{20} \mathrm{BrN}_{3} \mathrm{O}_{5} \mathrm{~S} \\
566\end{array}$ & $\begin{array}{l}260-262 \\
\text { Yellow }\end{array}$ & 65 \\
\hline 9 & $\begin{array}{c}\mathrm{C}_{26} \mathrm{H}_{21} \mathrm{BrN}_{2} \mathrm{O}_{3} \mathrm{~S} \\
521 \\
\end{array}$ & $\begin{array}{c}234-236 \\
\text { Orange }\end{array}$ & 75 \\
\hline 10 & $\begin{array}{c}\mathrm{C}_{28} \mathrm{H}_{25} \mathrm{BrN}_{2} \mathrm{O}_{4} \mathrm{~S} \\
565\end{array}$ & $\begin{array}{c}240-242 \\
\text { Orange }\end{array}$ & 80 \\
\hline 11 & $\begin{array}{c}\mathrm{C}_{26} \mathrm{H}_{20} \mathrm{BrN}_{3} \mathrm{O}_{5} \mathrm{~S} \\
566\end{array}$ & $\begin{array}{c}213-215 \\
\text { Orange }\end{array}$ & 75 \\
\hline 12 & $\begin{array}{c}\mathrm{C}_{32} \mathrm{H}_{23} \mathrm{BrN}_{6} \mathrm{O}_{7} \mathrm{~S} \\
731 \\
\end{array}$ & $\begin{array}{c}215-217 \\
\text { Orange }\end{array}$ & 65 \\
\hline 13 & $\begin{array}{c}\mathrm{C}_{32} \mathrm{H}_{23} \mathrm{BrN}_{6} \mathrm{O}_{7} \mathrm{~S} \\
731\end{array}$ & $\begin{array}{l}218-220 \\
\text { Yellow }\end{array}$ & 51 \\
\hline 14 & $\begin{array}{c}\mathrm{C}_{32} \mathrm{H}_{24} \mathrm{BrN}_{5} \mathrm{O}_{6} \mathrm{~S} \\
686\end{array}$ & $\begin{array}{l}207-209 \\
\text { Yellow }\end{array}$ & 55 \\
\hline 15 & $\begin{array}{c}\mathrm{C}_{34} \mathrm{H}_{28} \mathrm{BrN}_{5} \mathrm{O}_{7} \mathrm{~S} \\
730\end{array}$ & $\begin{array}{c}120-122 \\
\text { Yellow }\end{array}$ & 69 \\
\hline 16 & $\begin{array}{c}\mathrm{C}_{32} \mathrm{H}_{23} \mathrm{BrN}_{6} \mathrm{O}_{8} \mathrm{~S} \\
731\end{array}$ & $\begin{array}{c}239-240 \\
\text { Orange }\end{array}$ & 71 \\
\hline 17 & $\begin{array}{c}\mathrm{C}_{40} \mathrm{H}_{27} \mathrm{BrN}_{6} \mathrm{O}_{11} \mathrm{~S} \\
879\end{array}$ & $\begin{array}{l}208-210 \\
\text { Yellow }\end{array}$ & 63 \\
\hline 18 & $\begin{array}{c}\mathrm{C}_{40} \mathrm{H}_{27} \mathrm{BrN}_{6} \mathrm{O}_{11} \mathrm{~S} \\
879\end{array}$ & $\begin{array}{l}244-246 \\
\text { Yellow }\end{array}$ & 62 \\
\hline 19 & $\begin{array}{c}\mathrm{C}_{40} \mathrm{H}_{28} \mathrm{BrN}_{5} \mathrm{O}_{9} \mathrm{~S} \\
834 \\
\end{array}$ & $\begin{array}{l}189-191 \\
\text { Yellow }\end{array}$ & 60 \\
\hline 20 & $\begin{array}{c}\mathrm{C}_{42} \mathrm{H}_{32} \mathrm{BrN}_{5} \mathrm{O}_{10} \mathrm{~S} \\
878\end{array}$ & $\begin{array}{c}147-149 \\
\text { Orange }\end{array}$ & 55 \\
\hline 21 & $\begin{array}{c}\mathrm{C}_{40} \mathrm{H}_{27} \mathrm{BrN}_{6} \mathrm{O}_{11} \mathrm{~S} \\
879\end{array}$ & $\begin{array}{l}267-278 \\
\text { Yellow }\end{array}$ & 70 \\
\hline
\end{tabular}




\section{Results and Discussions.}

Formation of new $\beta$-amino Ketones compound (7-11)[20] Were obtained from the reaction of compound (1) and chalcones (2-6), which is shown in the following mechanism. Then Schiff bases (12-16) are prepared by the reaction of compounds (7-11) with 2,4-dinitro aniline in abs. $\mathrm{EtOH}$, using glacial acetic acid as catalyst. When Schiff bases reacted with phthalic anhydride presence abs. EtOH, as a solvent, produces seven membered heterocyclic rings (cyclic addition reaction)[21]. All the spectrum data were involved in table (2).

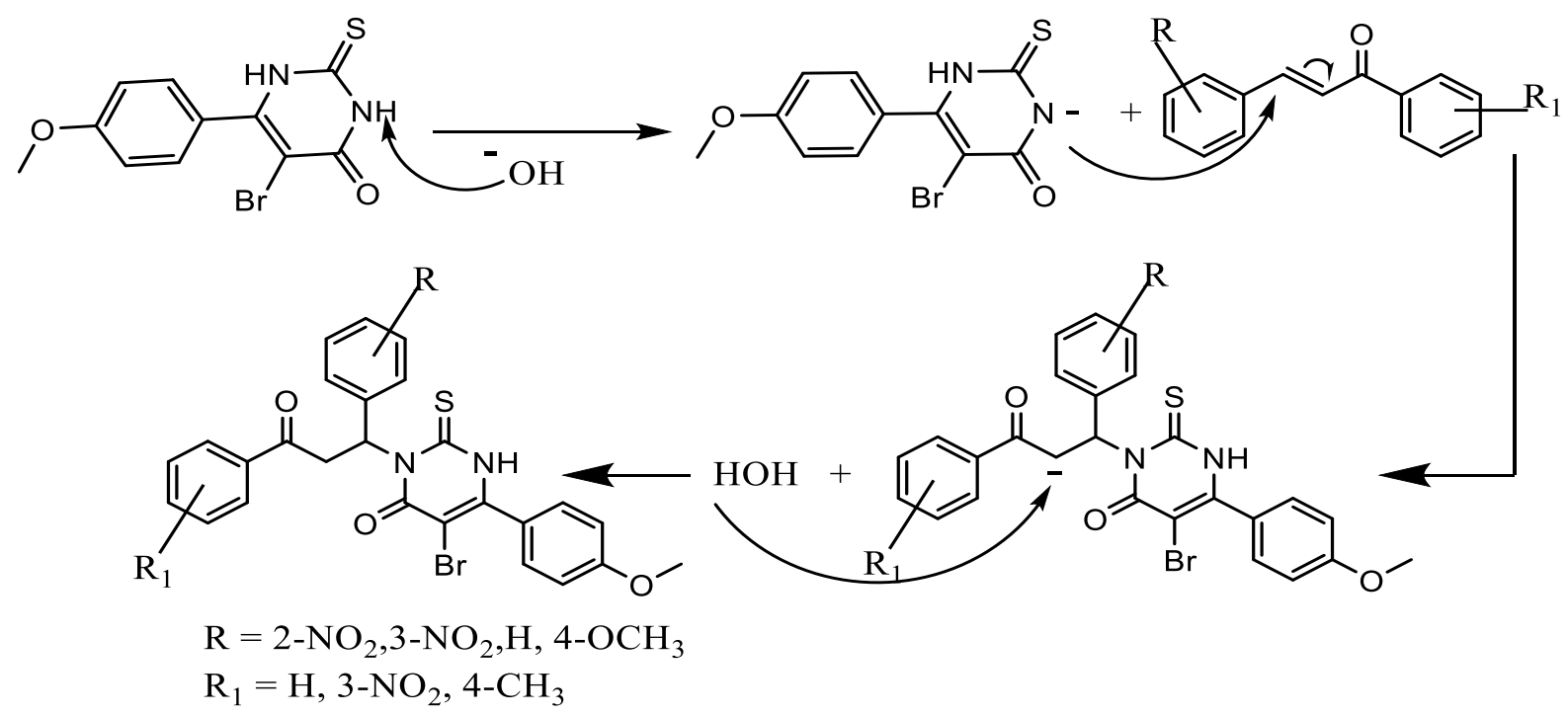

Figure1: Mechanism of syntheses $\beta$-amino ketones compound (7-11) 
Journal of Education and Science (ISSN 1812-125X), Vol: 29, No: 1, 2020 (218-232)

Table (2) Some spectral data for compounds (1-10)

\begin{tabular}{|c|c|c|c|c|c|c|}
\hline \multirow{2}{*}{$\begin{array}{l}\text { Comp. } \\
\text { No }\end{array}$} & \multirow{2}{*}{$1 \mathrm{HNMR}$} & \multicolumn{5}{|c|}{$\mathrm{IR} v \mathrm{~cm}^{-1}$} \\
\hline & & $\mathrm{N}-\mathrm{H}$ & Aro & Alp & $\begin{array}{l}\mathrm{C}=\mathrm{O} \\
\text { amide }\end{array}$ & Other \\
\hline $1 *$ & $\begin{array}{l}\delta: 3.82\left(\mathrm{~s}, 3 \mathrm{H}, \mathrm{OCH}_{3}\right), 7.09-7.55(\mathrm{~d}, 4 \mathrm{H}, \mathrm{ph}), \\
9.03(\mathrm{~s}, 1 \mathrm{H}, \mathrm{NH}),, 9.34(\mathrm{~s}, 1 \mathrm{H}, \mathrm{NH})\end{array}$ & 3195 & 3058 & 2981 & 1662 & $\begin{array}{l}\text { Ar-C=C (1600-1444) } \\
C=S(1251) \\
\text { (Asy, C-O-C) (1145) } \\
\text { (Sym, C-O-C) (1076) } \\
\text { C-Br (746) }\end{array}$ \\
\hline 2 & 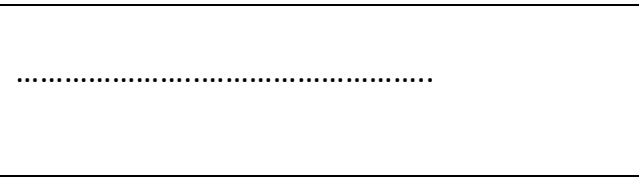 & ........... & 3040 & ........... & $\begin{array}{l}1662 \\
\text { keton }\end{array}$ & $\begin{array}{l}\mathrm{C}=\mathrm{C}(1606) \\
\mathrm{Ar}-\mathrm{C}=\mathrm{C}(1483) \\
\left(\text { Asy, } \mathrm{NO}_{2}\right)(1571) \\
(\text { Sym, NO} \\
\left.\mathrm{NO}_{2}\right)(1348)\end{array}$ \\
\hline 3 & 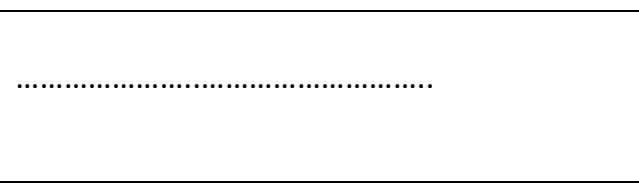 & ........... & 3033 & ........... & $\begin{array}{l}1662 \\
\text { keton }\end{array}$ & $\begin{array}{l}\mathrm{C}=\mathrm{C}(1604) \\
\mathrm{Ar}-\mathrm{C}=\mathrm{C}(1442) \\
\left(\text { Asy, } \mathrm{NO}_{2}\right)(1525) \\
\left(\mathrm{Sym}, \mathrm{NO}_{2}\right)(1340)\end{array}$ \\
\hline 4 & 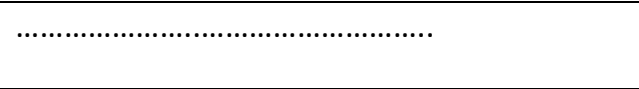 & $\ldots \ldots \ldots$ & 3061 & $\ldots \ldots \ldots$ & $\begin{array}{l}1663 \\
\text { Keton }\end{array}$ & $\begin{array}{l}C=C(1595) \\
A r-C=C(1415)\end{array}$ \\
\hline 5 & (n)......................... & ........... & 3001 & 2972 & $\begin{array}{l}1654 \\
\text { Keton }\end{array}$ & $\begin{array}{l}\text { Ar-C=C (1454) } \\
C=C(1602) \\
(\text { Asy, C-O-C) (1168) } \\
(\text { Sym,C-O-C) (1029) }\end{array}$ \\
\hline 6 & $\ldots$ & .......... & $\ldots \ldots \ldots$ & 2873 & $\begin{array}{l}1650 \\
\text { Keton }\end{array}$ & $\begin{array}{l}\mathrm{Ar}-\mathrm{C}=\mathrm{C}(1422) \\
\left(\text { Asy, } \mathrm{NO}_{2}\right) \quad(1562) \\
\left(\mathrm{Sym}, \mathrm{NO}_{2}\right)(1342)\end{array}$ \\
\hline 7 & $\ldots$ & 3390 & 3105 & 2848 & 1665 & $\begin{array}{l}\text { (C=O, Ketones) (1708) } \\
\left(\text { Asy, } \mathrm{NO}_{2}\right) \quad(1539) \\
\left(\mathrm{Sym}, \mathrm{NO}_{2}\right)(1345) \\
(\mathrm{C}=\mathrm{S})(1195) \\
(\text { Asy, C-O-C) }(1103) \\
(\mathrm{Sym}, \mathrm{C}-\mathrm{O}-\mathrm{C})(1004) \\
(\mathrm{C}-\mathrm{Br})(736)\end{array}$ \\
\hline $8^{*}$ & $\begin{array}{l}\delta: \\
\left.\text { 3.33(s-distroted, } 3 \mathrm{H}, \mathrm{CHCH}_{2}\right), \\
\text { 3.811(s,3H,OCH}{ }_{3}, \\
\text { 7.10-8.23(m,13H,Ar-H),8.79(s, NH) }\end{array}$ & 3209 & 3080 & 2962 & 1666 & $\begin{array}{l}\text { (C=O, Ketones) (1691) } \\
(\text { Asy, NO } 2) \\
(1527,1508) \\
(\text { Sym, NO })(1348) \\
(\mathrm{C}=\mathrm{S})(1249) \\
(\text { Asy, C-O-C) }(1031) \\
(\text { Sym,C-O-C) }(1008) \\
(\text { C-Br) } 684)\end{array}$ \\
\hline $9 * *$ & $\begin{array}{l}\delta: \\
2.60\left(\mathrm{~s}-\text { distroted }, 3 \mathrm{H}, \mathrm{CHCH}_{2}\right) \\
3.88\left(\mathrm{~s}, 3 \mathrm{H}, \mathrm{OCH}_{3}\right), \\
6.98-7.93(\mathrm{~m}, 14 \mathrm{H}, \mathrm{Ar}), 9.879(\mathrm{~s}, 1 \mathrm{H}, \mathrm{NH})\end{array}$ & 3199 & 3010 & 2937 & 1662 & $\begin{array}{l}\text { (C=O, Ketones) (1729) } \\
(\mathrm{C}=\mathrm{S})(1251) \\
(\text { Asy, C-O-C) }(1103) \\
(\text { Sym,C-O-C) }(1006) \\
(\mathrm{C}-\mathrm{Br})(702)\end{array}$ \\
\hline $10 *$ & $\begin{array}{l}\delta: \\
2.61\left(\mathrm{~s}, 3 \mathrm{H}, \mathrm{CH}_{3}\right), 3.45\left(\mathrm{~s}-\text { distroted, } 3 \mathrm{H}, \mathrm{CHCH}_{2}\right), \\
3.91\left(\mathrm{~s}, 3 \mathrm{H}, \mathrm{OCH}_{3}\right), 7.02-8,06(\mathrm{~m}, 12 \mathrm{H}, \mathrm{Ar}-\mathrm{H}), \\
9.33(\mathrm{~s}, 1 \mathrm{H}, \mathrm{NH})\end{array}$ & 3210 & 3001 & 2972 & 1656 & $\begin{array}{l}\text { (C=O, Ketones) (1690) } \\
(\mathrm{C}=\mathrm{S})(1253) \\
(\text { Asy, C-O-C) (1170) } \\
(\text { Sym, C-O-C) (1033) } \\
(\mathrm{C}-\mathrm{Br})(676)\end{array}$ \\
\hline
\end{tabular}


Table (2) Some spectral data for compounds (11-21)

\begin{tabular}{|c|c|c|c|c|c|c|}
\hline $11^{* *}$ & $\begin{array}{l}\delta: \\
1.53\left(\mathrm{br}, 2 \mathrm{H}, \mathrm{CH}_{2}\right), 2.1\left(\mathrm{~s}, 2 \mathrm{H}, \mathrm{CH}_{2}\right), \\
3.82\left(\mathrm{~s}, 3 \mathrm{H}, \mathrm{OCH}_{3}\right), \\
6.88-8.18(\mathrm{~m}, 13 \mathrm{H}, \mathrm{Ar}) \\
\text { 9.07( }, 1,1 \mathrm{H}, \mathrm{NH})\end{array}$ & 3211 & 3005 & 2995 & 1668 & $\begin{array}{c}\text { (C=O, Ketones) (1705) } \\
\left(\text { Asy, } \mathrm{NO}_{2}\right) \quad(1508) \\
\left(\text { Sym, } \mathrm{NO}_{2}\right)(1373) \\
(\mathrm{C}=\mathrm{S})(1209) \\
(\text { Asy, C-O-C) (1029) } \\
(\text { Sym,C-O-C) (1004) } \\
\text { (C-Br) (721) }\end{array}$ \\
\hline $12 *$ & $\begin{array}{l}\delta: \\
3.33(\mathrm{~s}, 1 \mathrm{H}, \mathrm{CH}), 3.81\left(\mathrm{~s}, \mathrm{OCH}_{3}\right), \\
5.80\left(\mathrm{~s}, \mathrm{CH}_{2}\right), 6.94-8.21(\mathrm{M}, 8 \mathrm{H}, \mathrm{Ar}-\mathrm{H}), \\
9.09(\mathrm{~s}, 1 \mathrm{H}, \mathrm{NH})\end{array}$ & 3193 & 3006 & 2985 & 1666 & $(C=N)(1602)$ \\
\hline 13 & , & 3202 & 3030 & 2945 & 1666 & $(C=N)(1602)$ \\
\hline 14 & , & 3197 & 3001 & 2960 & 1670 & $(C=N)(1629)$ \\
\hline 15 & 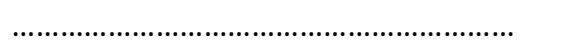 & 3336 & 3052 & 2933 & 1654 & $(C=N)(1630)$ \\
\hline 16 & 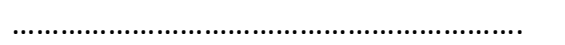 & 3205 & ............ & 2997 & 1668 & $(C=N)(1631)$ \\
\hline $17^{*}$ & $\begin{array}{l}\delta: 3.34\left(\mathrm{~s} \text {-distroted, } 3 \mathrm{H}, \mathrm{CHCH}_{2}\right) \\
3.81\left(\mathrm{~s}, 3 \mathrm{H}, \mathrm{OCH}_{3}\right), \\
7.08-8.17(\mathrm{~m}, 2 \mathrm{OH}, \mathrm{Ar}), 9.09(\mathrm{~s}, 1 \mathrm{H}, \mathrm{NH})\end{array}$ & 3194 & 3001 & 2961 & 1663 & $(\mathrm{C}=\mathrm{O}$, lacton) $(1715)$ \\
\hline 18 & 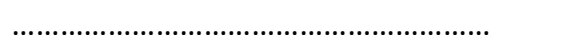 & 3193 & 3010 & 2951 & 1663 & $(\mathrm{C}=\mathrm{O}$, lacton $)(1705)$ \\
\hline $19 * * *$ & $\begin{array}{l}\delta: 2.87\left(\mathrm{br}, 3 \mathrm{H}, \mathrm{CHCH}_{2}\right), 3.82\left(\mathrm{~s}, 3 \mathrm{H}, \mathrm{OCH}_{3}\right), \\
7.22-8.18(\mathrm{~m}, 21 \mathrm{H}, \mathrm{Ar}), 8.92(\mathrm{~s}, 1 \mathrm{H}, \mathrm{NH})\end{array}$ & 3195 & 3043 & 2952 & 1663 & $(\mathrm{C}=\mathrm{O}$, lacton) $(1702)$ \\
\hline 20 & 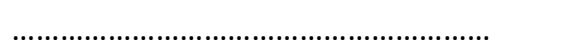 & 3205 & 3008 & 2924 & 1639 & $(\mathrm{C}=\mathrm{O}$, lacton $)(1700)$ \\
\hline $21^{*}$ & $\begin{array}{l}\delta: 3.33\left(\mathrm{br}, 3 \mathrm{H}, \mathrm{CHCH}_{2}\right), 3.81\left(\mathrm{~s}, 3 \mathrm{H}, \mathrm{OCH}_{3}\right) \text {, } \\
7.08-8.83(\mathrm{~m}, 2 \mathrm{OH}, \mathrm{Ar}), 9.09(\mathrm{~s}, 1 \mathrm{H}, \mathrm{NH})\end{array}$ & 3184 & 3002 & 2941 & 1662 & $(\mathrm{C}=0$, lacton) (1716s) \\
\hline
\end{tabular}

$*($ solvent, DMSO $), * *\left(\right.$ solvent, $\left.\mathrm{CDCL}_{3}\right), * * *\left(\right.$ solvent, Aceton- $\left.\mathrm{d}_{6}\right)$ 


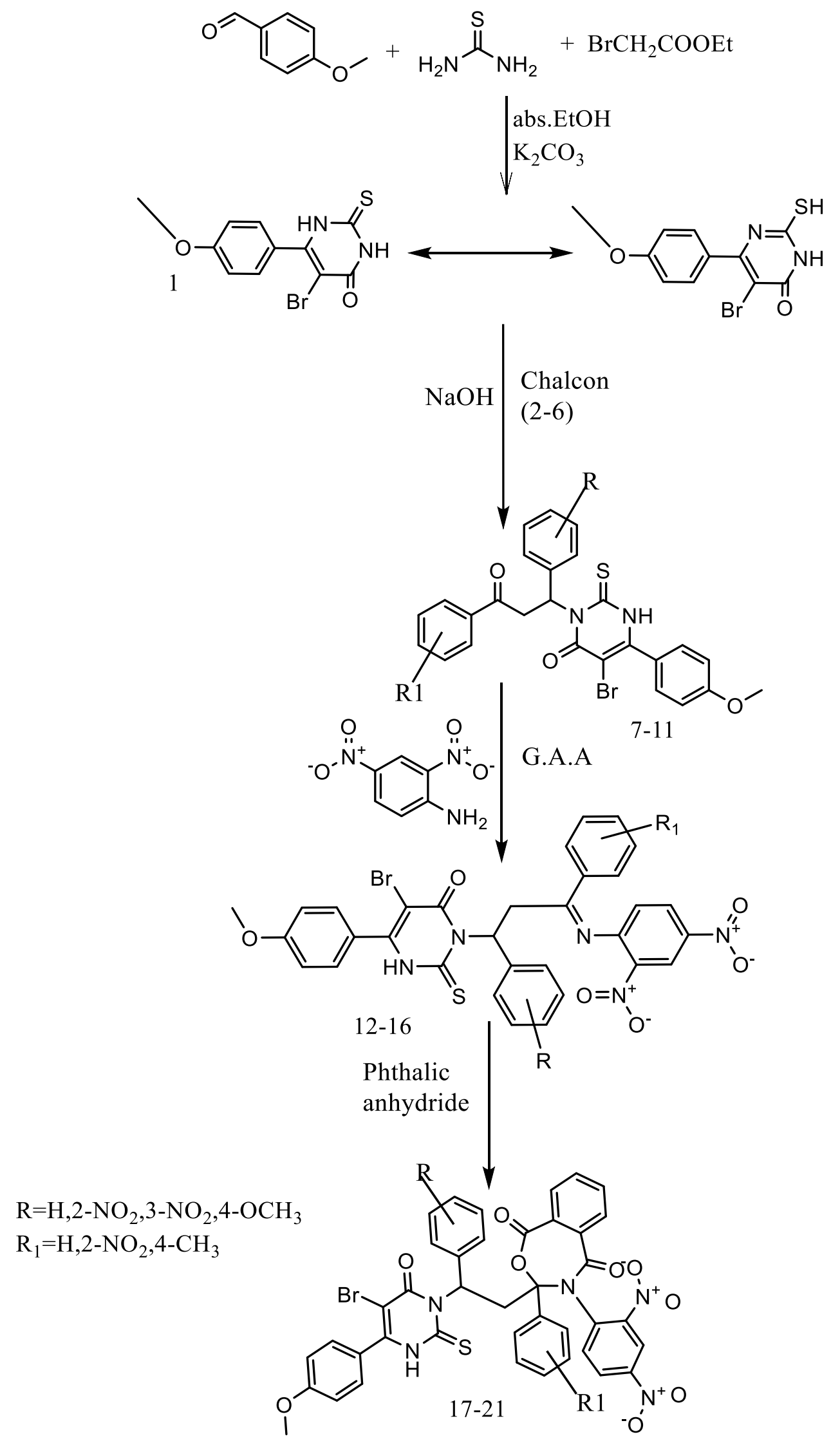

Scheme (1) illustrates the prepared compounds (1-21) 


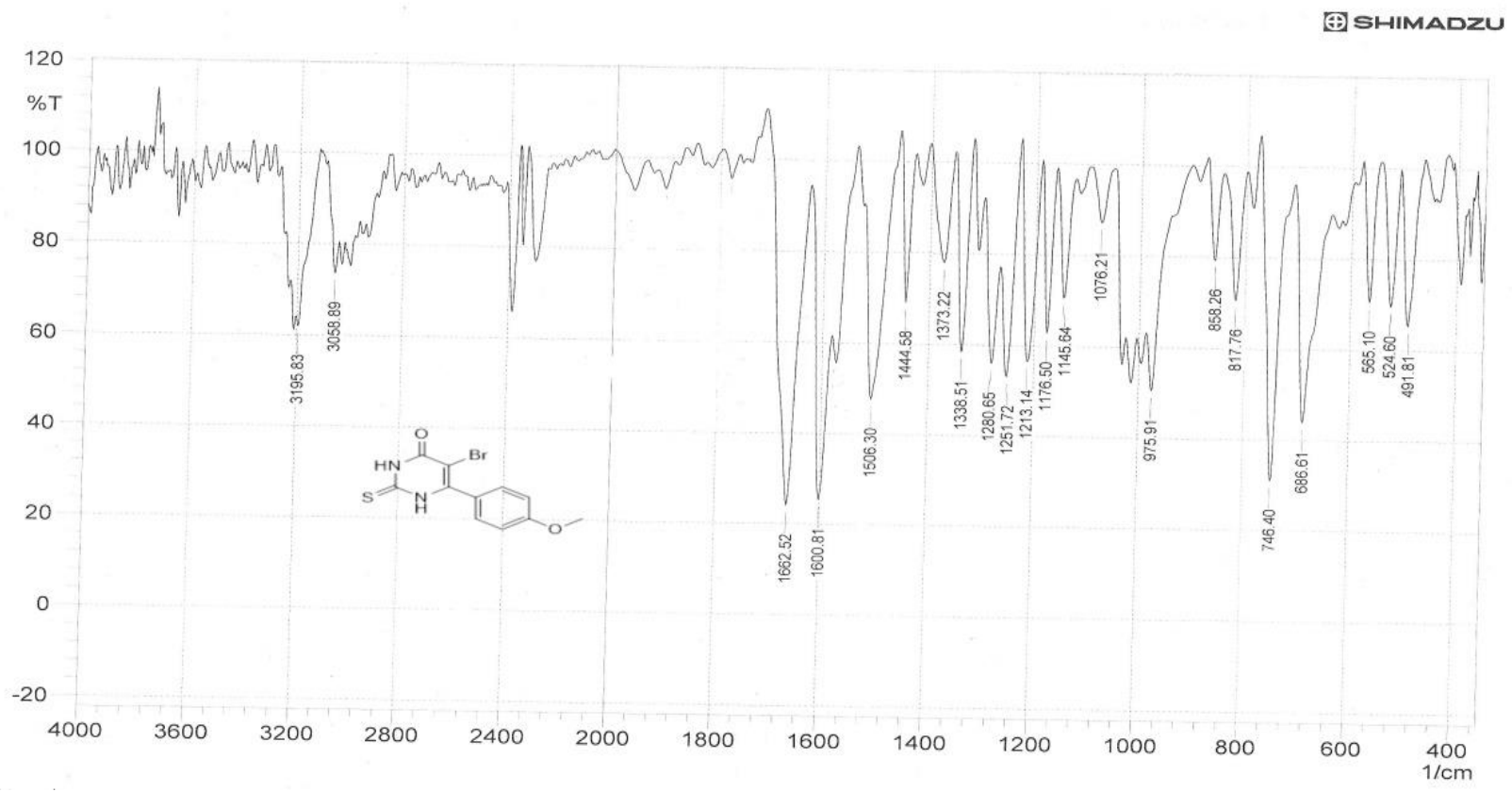

Figure2: FT-IR For- comp: (1)

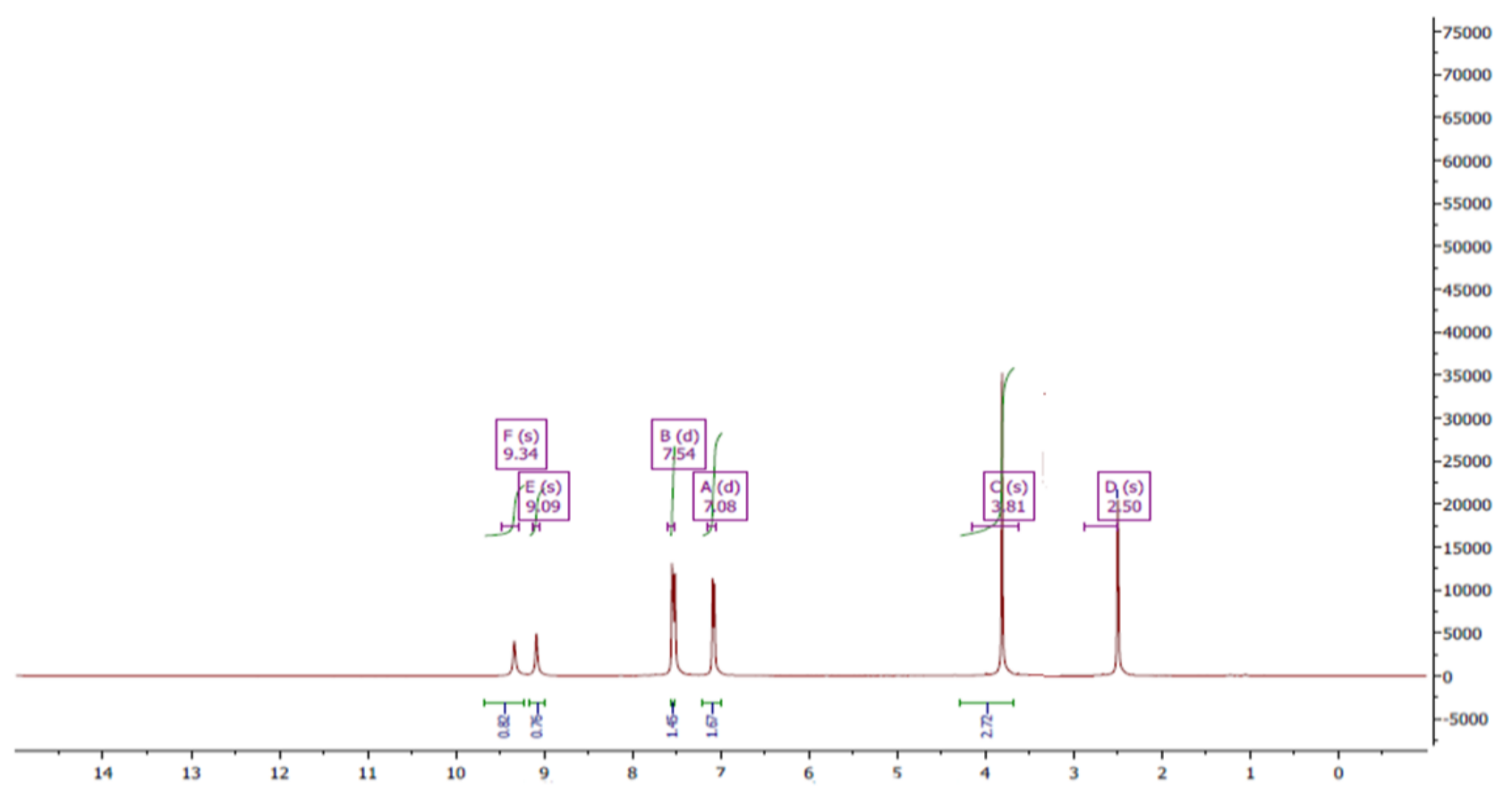

Figure3: 1HNMR For- comp: (1) 
๑ SHIMADZU

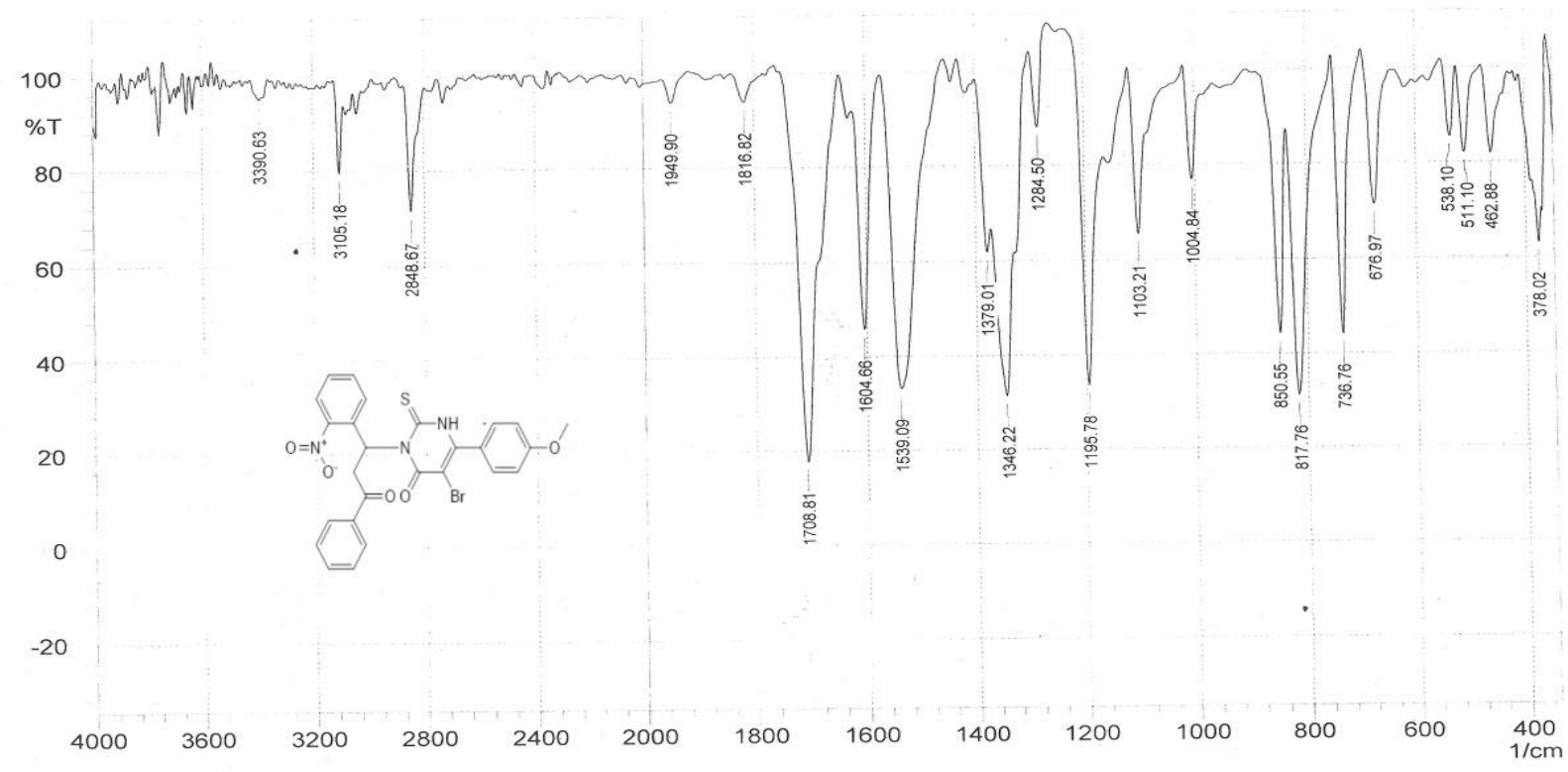

Figure4: FT-IR For- comp: (7)

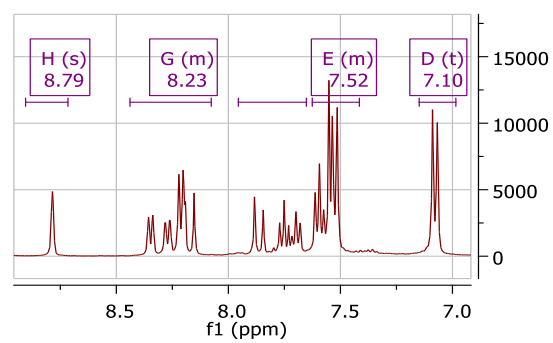

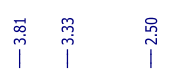
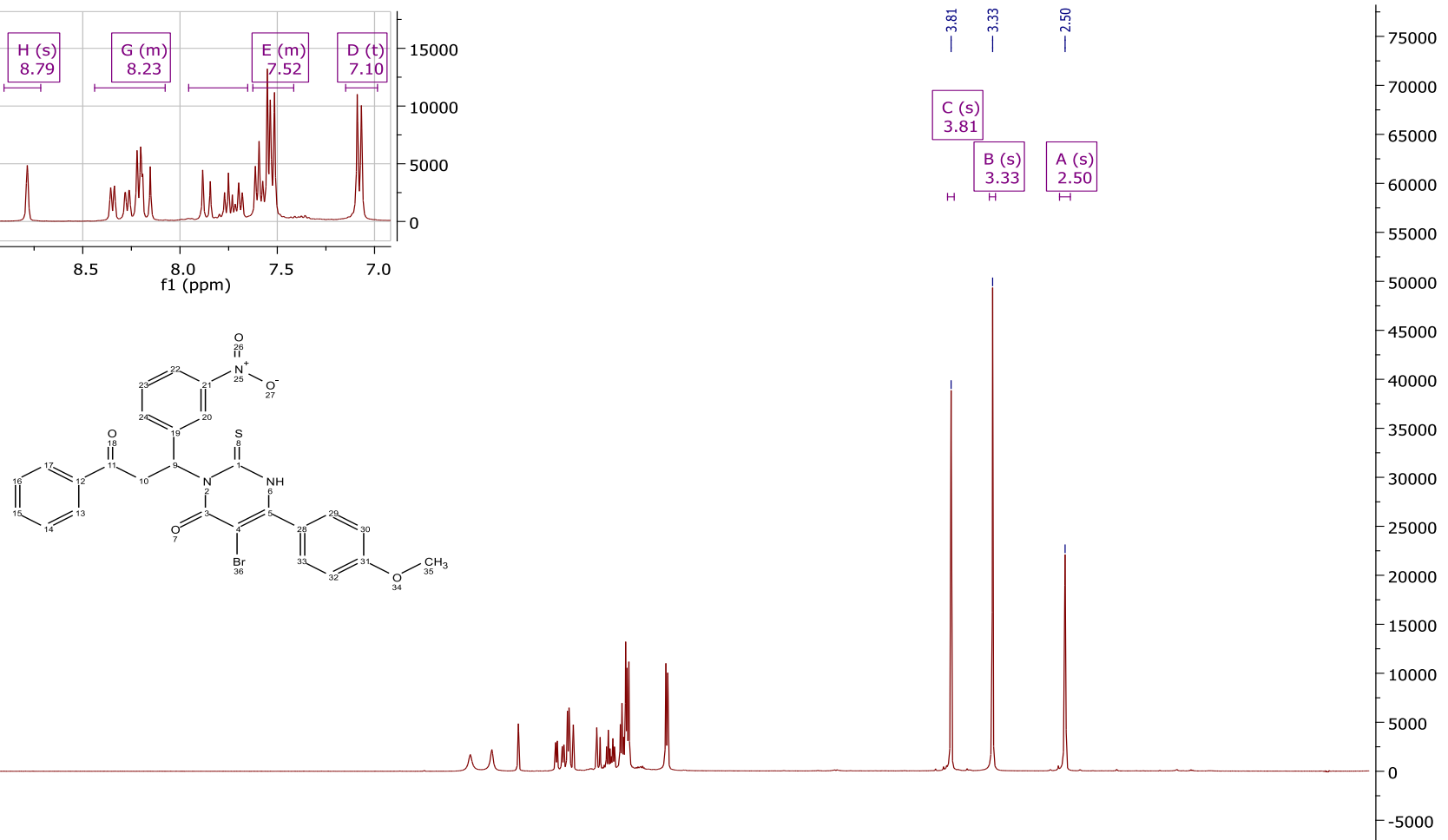

14

13
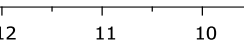

$\Lambda$
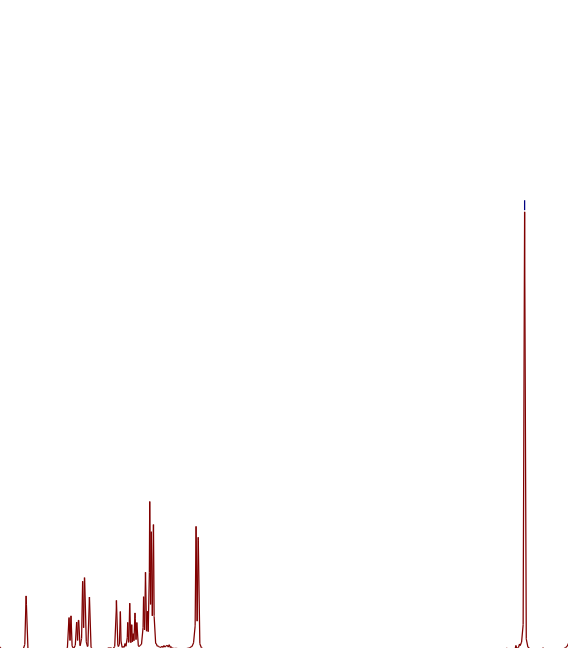

Figure5: 1HNMR For- comp: (8) 


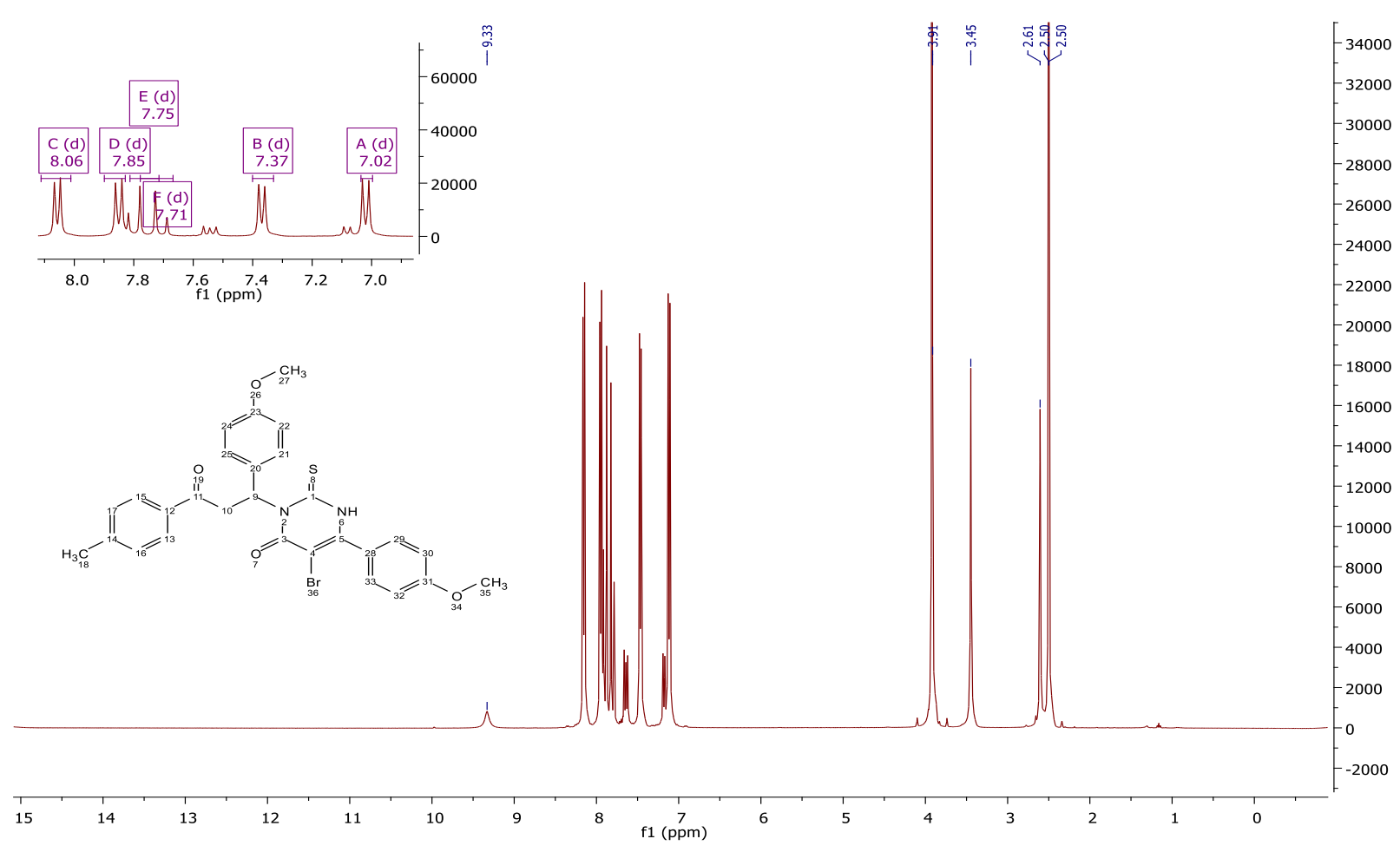

Figure6: 1HNMR For- comp: (10)
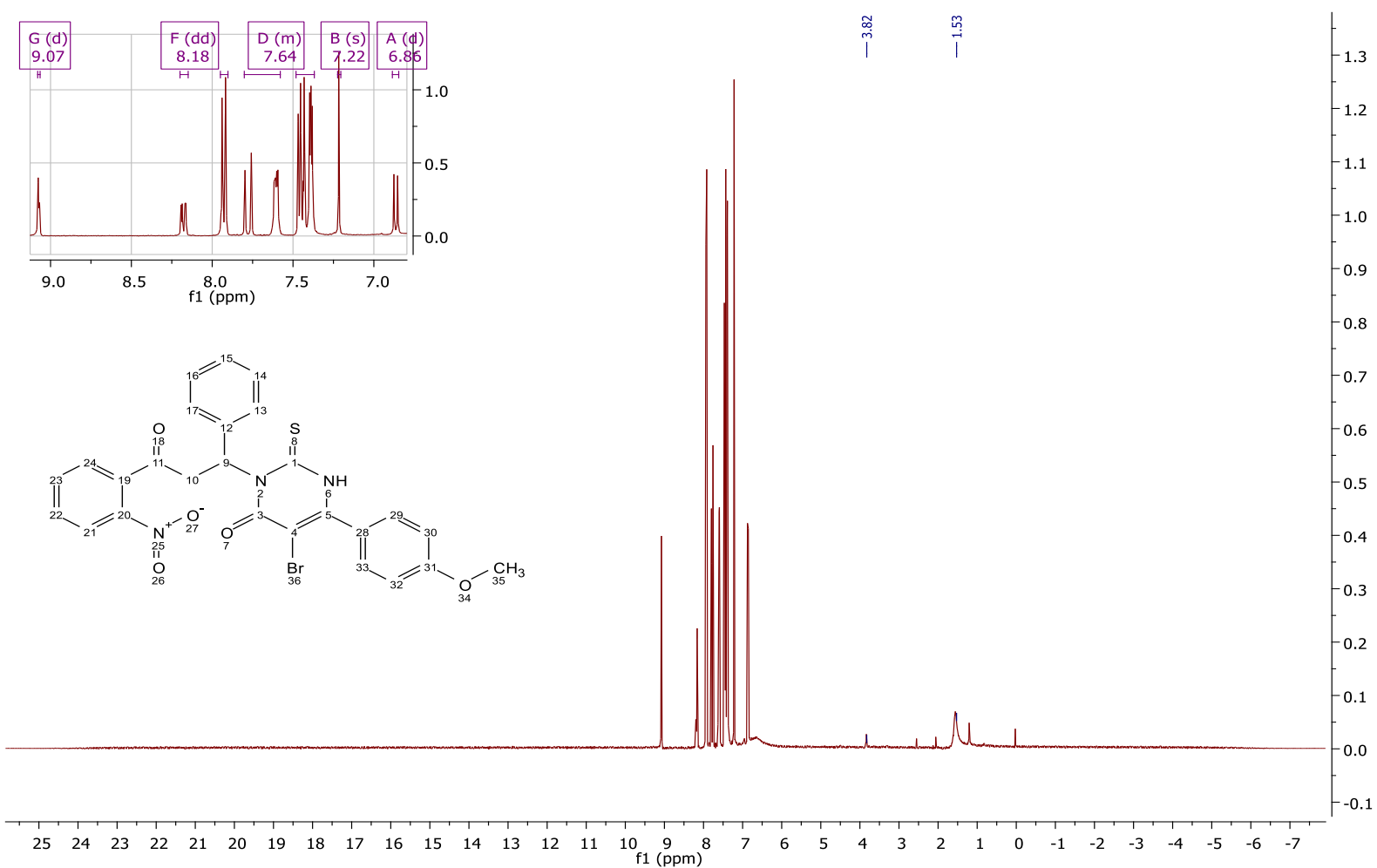

Figure7: 1HNMR For- comp: (11) 
ФSHIMADZU

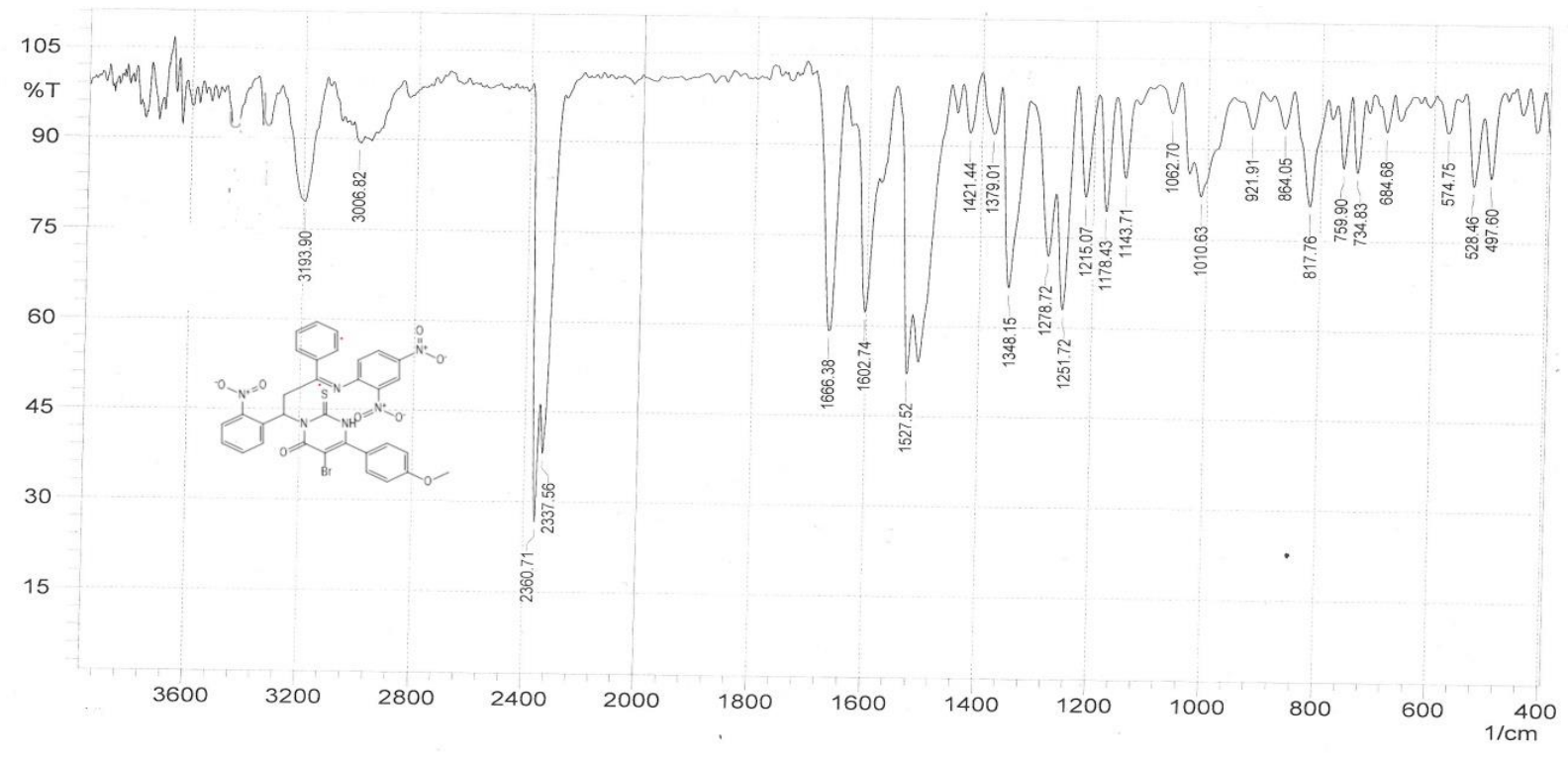

Figure8: FT-IR For- comp: (12)
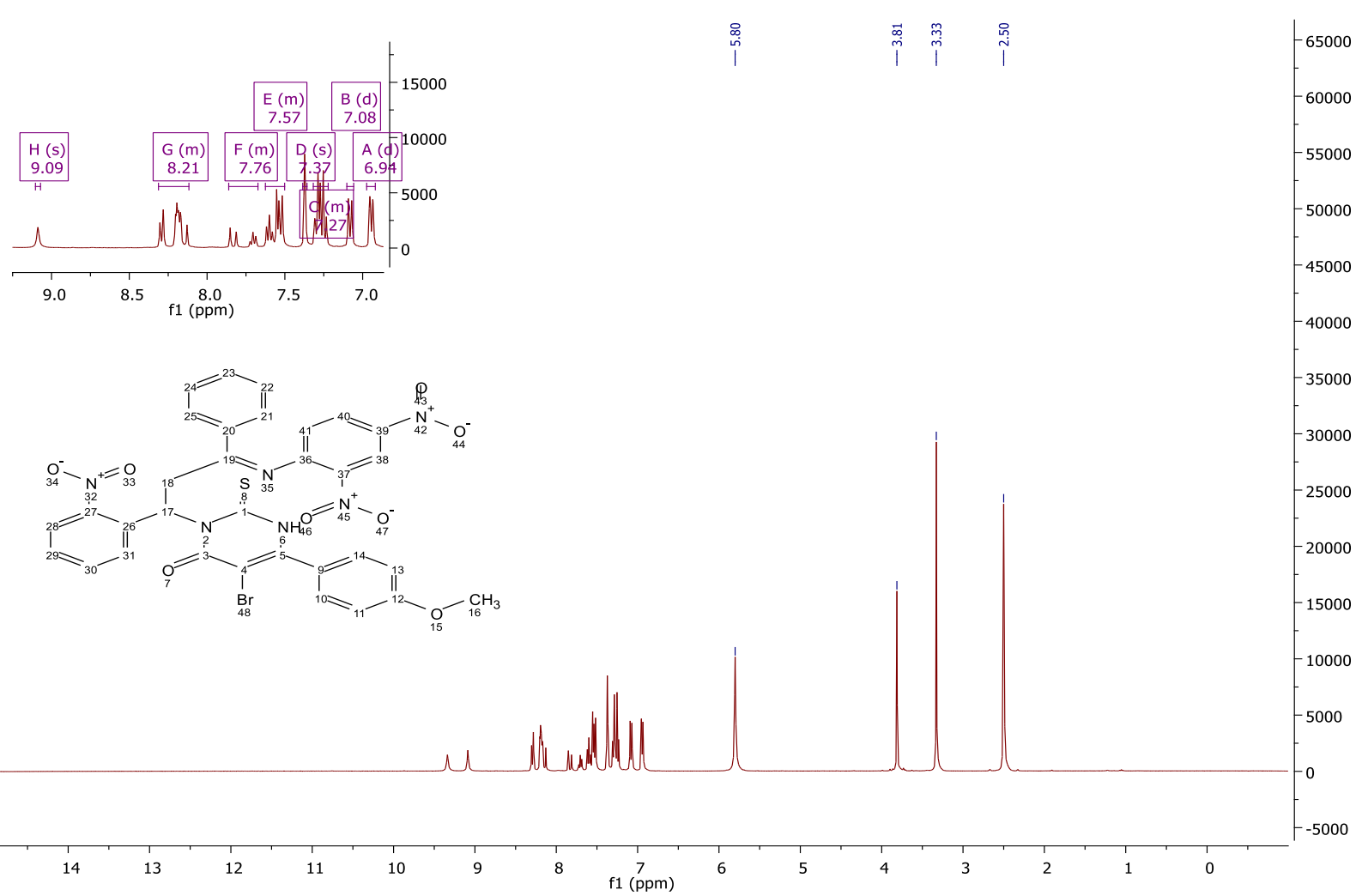

Figure9: 1HNMR For- comp: (12) 


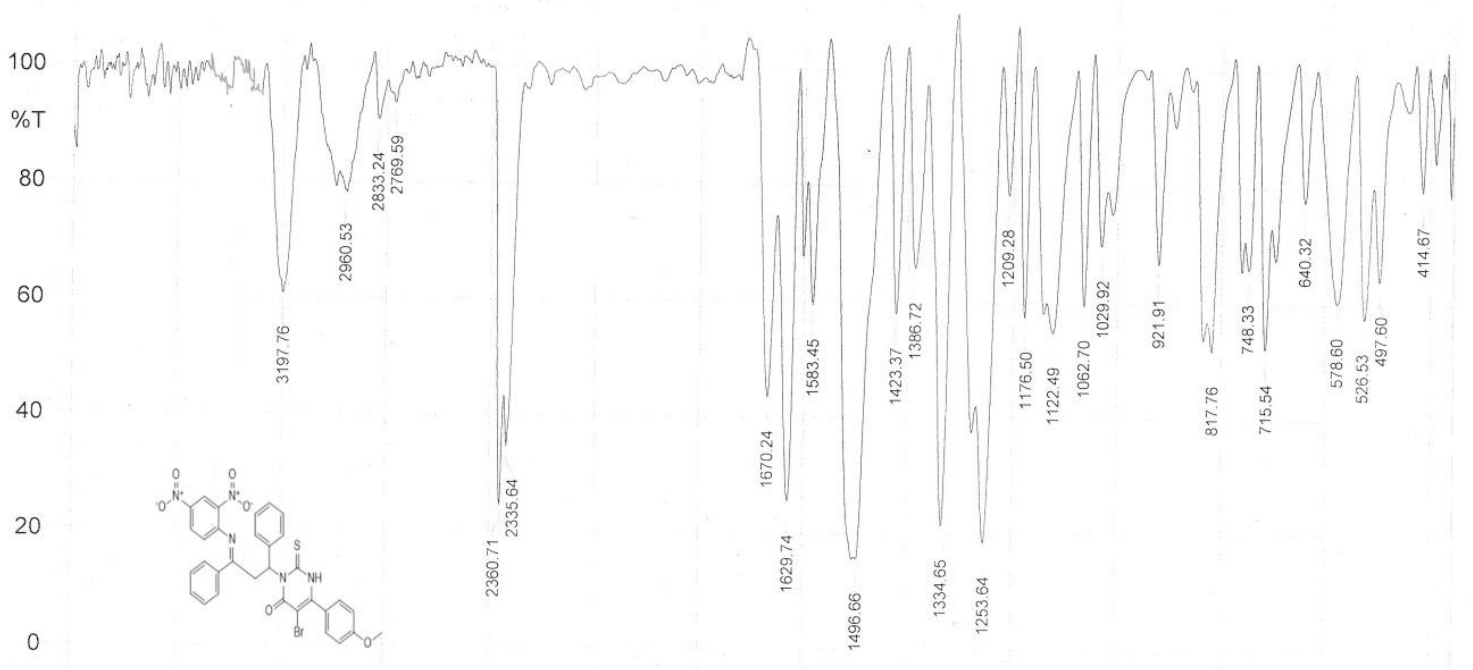

Figure10: FT-IR for comp. (14)
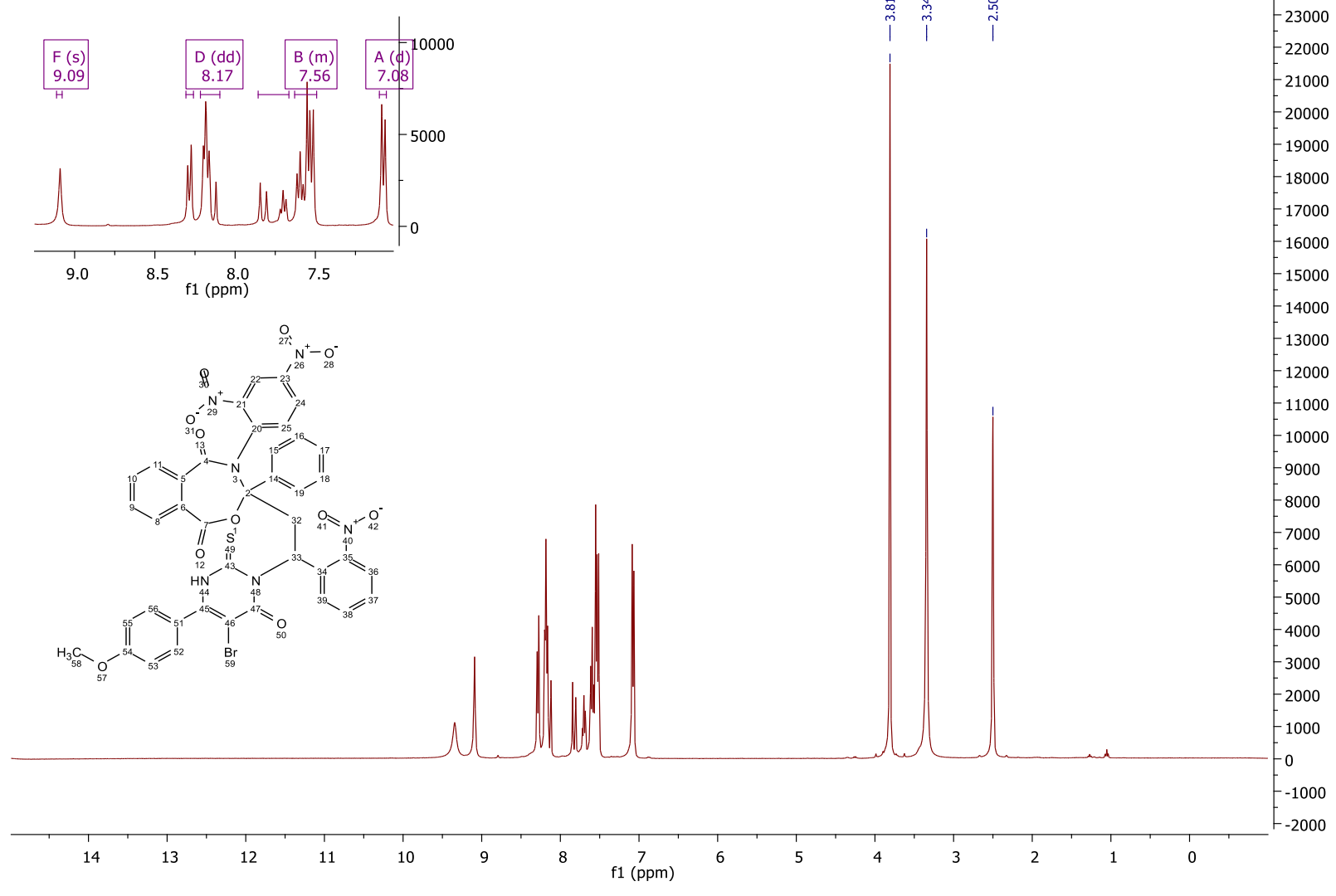

Figure11: 1HNMR For- comp: (17) 


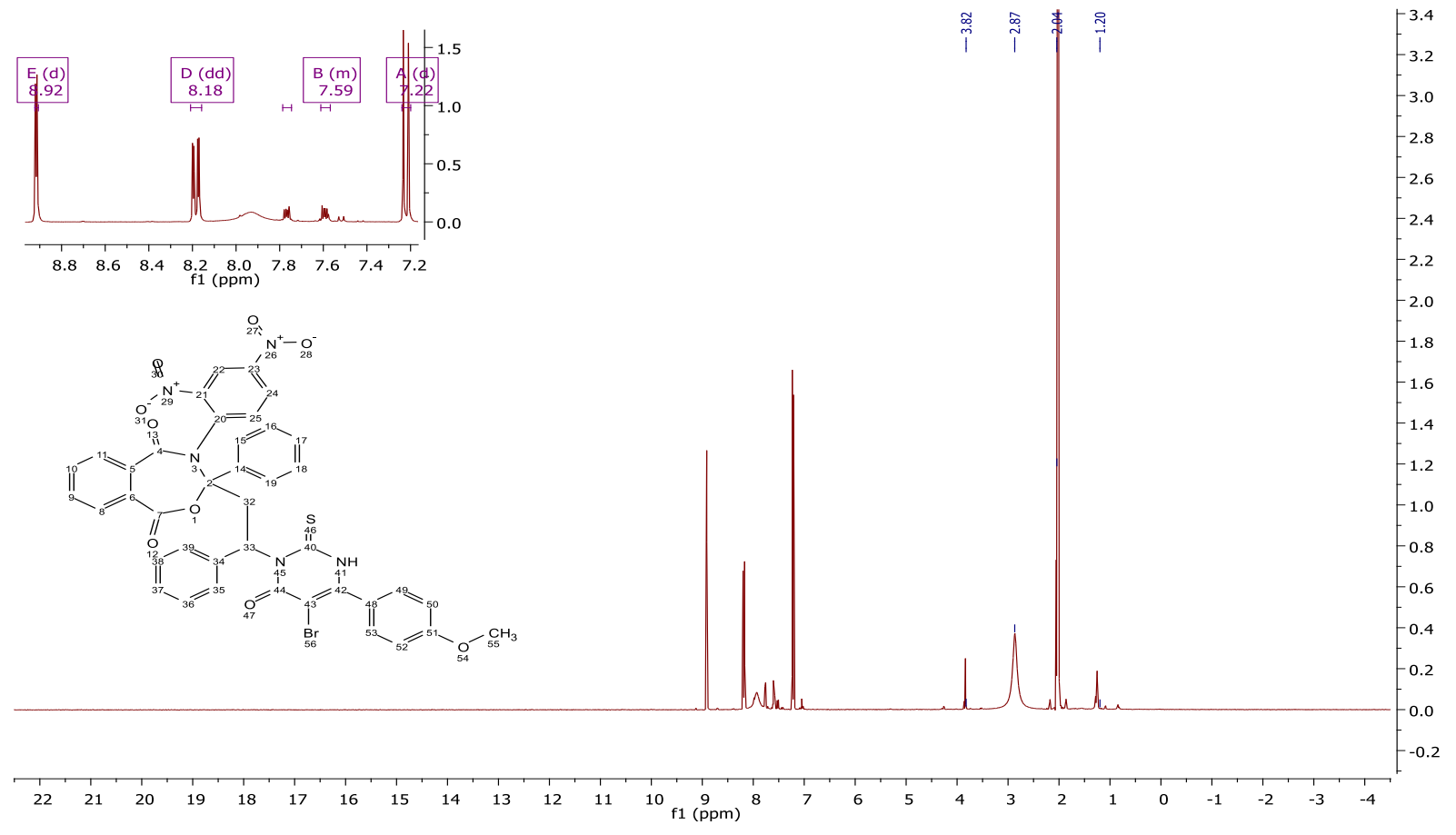

Figure12: 1HNMR For- comp: (19)

\section{Ankowledgement}

We are grateful to Department of Chemistry, College of Education for Pure Science, Mosul University, for the Facilities given to perform this work.

\section{Reference}

1. Sasai T., Arai. T., Satow. Y., Houk. K. N., Shibasaki. M. J. Am., Chem. Soc., 117, 6194-6191, (1995).

2. Shibasaki M., Sasai. H \& Arai. T., Angew., Chem. Ed. Engl.,36, (1997).

3. Fernandez I., Araujoc. S., Romero. J., Alcudia. F., Khiar. N., Tetrahedron, 56,3749-3753, (2000).

4- Lee P. H. Ahn, H., Lee, K., Sung, S., Kim, S., Tetrahedron Lett., 42,37-39, (2001).

5- Shaikh N. S., Deshpande. V. H., BedeKar. A. V., Tetrahedron, 57, 9045, (2001). 
6- Cheng S., Cromer. D. D., Tetrahedron Lett., 43,1179-1181, (2001).

7- Aralmurugan S. K., Venkatraman. H. P., Rasayan. P. R., J. Chem.,3, 385, (2010).

8- Wadeher J. S., Puranik. P. M., Karande. A. N., Yeole. G. P., I. J. of Pharm. Tech. Research, pp.22-33., 1. (2009).

9- Desai S. B., Desai. P. B., Hetrocycl Common, pp. 83-90., 7(1). (2001).

10- Pathak P., Jolly. V. S., Sharma. K., Oriental J. Chem., pp.161-162, 16(1), (2000).

11- Samadhiya S., Halva. A., Oriental J. Chem., pp.119-122, 17(1),(2001).

12-Wilson O. C., Givold. O., "Text book of organic medicinal and pharmaceutical Chemistry", 5th. Ed, (1966).

13- Yass A. I., Kerbala J. of pharmaceutical science, p.49-58, (2010).

14- Mohammed J.M., Ahmad. Kh. A., Abachi. T. F., Tikrit J. of Pharmaceutical Sciences.12(1),76-89. (2017).

15- Furniss S. B., Hannaford. J. A., Smith. C. W. P., and Tatchell. R. A., Vogel's Text book of Practical Organic Chemistry, Fifth edition. 1023-1036. (1989).

16- Himaja M., Rout. K . P., Karigar. A. A., Daspoppy. D. Munirajsekhar, Universal, J.of Pharmacy, 1(1), 72-77, (2012).

17- Mohmed Z. Waffa. B., Bouchaib. B. Ahmed. R., Said. S., Arkivoc, 150-161, (2005).

18- Al-Rawi S. M., Ph.D. Thesis. Department of Chem., College of Education IbnAlHaitham, University of Baghdad. Baghdad, Iraq, (2011), (In Arbic)

19- Hallinan A. E., Hagen. J. T., Tsymbalov. S., Husa. K. R., Stapelfeld. C. A., Savage. A. ,M., J. Med. Chem. 39(2), 609-613, (1996).

20- Al-Jaber A.N., Bougasim. A. A., Karah. S. M., . J. of Saudi Chemical Society. 16, 
Journal of Education and Science (ISSN 1812-125X), Vol: 29, No: 1, 2020 (218-232)

45-53. 16, (2012).

21- Level A., Comm., Reg. Issue 42. 75s(9). 2187-2192, (2008). 